\title{
Unveiling the physics behind the spectral variations of changing-look quasars with optical polarimetry
}

\author{
F. Marin
}

\begin{abstract}
Université de Strasbourg, CNRS, Observatoire astronomique de Strasbourg, UMR 7550, 67000 Strasbourg, France e-mail: frederic.marin@astro.unistra.fr
\end{abstract}

Received 7 August 2017 / Accepted 6 September 2017

\begin{abstract}
A handful of active galactic nuclei (AGN) have shown strong spectral variations in the optical band between epochs that are years apart. The appearance or disappearance of broad emission lines in their spectra completely changes their classification. Since their nucleus orientation cannot change on such short timescales, another physical interpretation has to be found. Several scenarios have been offered to explain their changing-look nature, and for the first time, we conduct polarized radiative transfer Monte Carlo simulations for all the models. We demonstrate that all interpretations have distinctive features in both total optical flux and continuum polarization, such as suggested by pioneering previous observations. Distinguishing between the different scenarios is thus straightforward. We apply our results on the changing-look quasar J1011+5442 and confirm the conclusions found previously: in this specific case, the disappearance of the broad emission lines is due to a change in accretion rate.
\end{abstract}

Key words. galaxies: active - galaxies: Seyfert - polarization - radiative transfer - scattering

\section{Introduction}

Active galactic nuclei (AGN) are parsec-scale powerful lighthouses that reside in the core of galaxies. Their intrinsic luminosity often outshines the light from their host galaxy, and such an amount of radiation can only be produced by accretion onto a supermassive black hole (Pringle \& Rees 1972; Shakura \& Sunyaev 1973). This central engine irradiates its close environment, which in the first half parsec is mainly constituted of electrons and atoms. Through photoionization by continuum photons, emission lines emerge from the gas, which has a compact and equatorial morphology (Gaskell 2009). Submitted to Keplerian motion, this region naturally emits broadened lines. However, not all AGN show broad emission lines, which led to the classification of quasars ${ }^{1}$ according to the presence (type 1) or absence (type 2) of these specific spectral features (see, e.g., Rowan-Robinson 1977). We only realized that all AGN types might be correlated by their inclination when Antonucci \& Miller (1985) discovered broad emission lines in the polarized flux of a Seyfert type 2 galaxy. It follows that type 1 AGN are seen from the polar direction, where there is no equatorial obscuration, while type 2 AGN are seen from the equatorial plane. The broad line region (BLR) is thus hidden behind an opaque wall of dust for the latter type. The classification has several intermediate categories ranging from 1.2 to 1.8 , all showing broad emission lines, with an increasing [O III] $\lambda 5007 / \mathrm{H} \beta$ flux ratio (Osterbrock 1977). The last type, 1.9, includes AGN that only show broad $\mathrm{H} \alpha$ lines.

After it is optically classified, an AGN is not expected to flicker between two extreme types, at least not on a human timescale. Nevertheless, several "changing-look" AGN were discovered in the past decades. Examples of these AGN

\footnotetext{
1 "Quasar" is the historical term coined when the first quasi-stellar objects of this type were discovered. Unlike Seyfert galaxies, quasars are radio-loud, cosmological AGN situated in the distant Universe.
}

are Mrk 1018 (Cohen et al. 1986), NGC 1365 (Risaliti et al. 2000), SDSS J015957.64+003310.5 (LaMassa et al. 2015) or IC 751 (Ricci et al. 2016). These AGN are characterized by rapid(timescales of month to years) dimming and brightening in total flux, and they often show variation in the strength of their broad emission lines. In several cases, the broad emission disappears and an AGN changes from type 1 to type 1.9 or 2 (Cohen et al. 1986). The disappearance of the broad emission lines is often associated with a drop in the continuum flux, suggesting a common physical mechanism (LaMassa et al. 2015). Several scenarios are proposed to explain the optical-ultraviolet$\mathrm{X}$-ray variations. First is a possible obscuration of the central source by a large amount of dust or gas crossing the observer's line of sight. This could be due to the motion of dense cloudlets originating from the torus (e.g., Goodrich 1989) or a variation in the height of the circumnuclear dust region (e.g., Simpson 2005). This variation can occur after an peak in accretion activity, and the resulting intense radiation field wipes out the outer layers of dust. Conversely, the dusty torus can also recover from this activity and restore its amount of dust. A different interpretation was suggested by LaMassa et al. (2015) based on the pioneering work of Elitzur et al. (2014). In this scenario, a change in ionizing flux from the central engine, related to lower accretion activity, may cause the broad emission features and the BLR to disappear. A fainter continuum ultimately reduces the emission line intensity of the BLR since there are not enough photons to ionize the gas. The BLR (and later the torus) should disintegrate at low bolometric luminosities $\left(L_{\text {bol }} \leq 5 \times 10^{39} M_{7}^{2 / 3}\right.$, Elitzur \& Ho 2009) as a low accretion efficiency would not be able to sustain the required cloud flow rate (Elitzur \& Shlosman 2006; Elitzur et al. 2014). Understanding the physics behind the spectral variations of changing-look AGN is thus fundamental to probe the life cycle of galaxies, to determine how AGN evacuate or replenish their gaseous or dust material, and to verify the true morphology of the innermost AGN regions. 
To solve these questions, we present radiative transfer simulations of the polarized optical light emerging from those different scenarios. We aim to check whether optical polarimetry can unveil the true interpretation behind the type 1 to type 1.9/2 changing-look appearance of these peculiar AGN. In Sect. 2 we introduce the Monte Carlo code and the models to be investigated. We explore the total flux and polarimetric signal of the different models in Sect. 3 and apply our results to a specific object in Sect. 4. We conclude in Sect. 5 about the importance of polarimetry to unveil the correct case-by-case physical interpretation.

\section{Modeling changing-look AGN}

To model the optical continuum polarization expected from a changing-look AGN, we used the Monte Carlo radiative transfer code STOKES (Goosmann \& Gaskell 2007; Marin et al. 2012, 2015a). This numerical tool was used to model, predict, fit, and interpret the polarization signatures of a great variety of sources, from exoplanets to AGN (see, e.g., Marin \& Goosmann 2014; Marin \& Grosso 2017). STOKES works from the near-infrared to the hard X-ray bands and simulates the random and successive interactions of light with matter under a large number of possible geometries and scales. The code allows us to virtually explore all kinds of geometrical configurations for an AGN, from the central supermassive black hole to extended outflows. All the scattering physics is included in the code (see Goosmann \& Gaskell 2007), and the user can register the linear and circular polarization at all polar and azimuthal viewing angles. The code, in its basic version, is available online ${ }^{2}$. It is the same version of STOKES that was used in this publication.

Our basic AGN model is presented in Fig. 1 (unscaled). The model consists of a central point-like isotropic monoenergetic source that irradiates $\lambda 550 \mathrm{~nm}$ photons. We selected this wavelength according to the effective wavelength midpoint for a standard $V$ filter (Binney \& Merrifield 1998). This is also the waveband in which very many historical AGN polarization measures were achieved (see, e.g., Brindle et al. 1990; Smith et al. 2002). Because the scattering-induced polarization of AGN is almost-wavelength independent in the ultraviolet, optical, and near-infrared bands (Miller \& Antonucci 1983; Code et al. 1993; Smith et al. 1997), our modeling is conservative for broadband observations. Only the amount of depolarization from external sources can vary with wavelength, allowing for easier polarimetric detections in the ultraviolet waveband where the host contamination is weaker (Zirbel \& Baum 1998).

At a distance of $0.01 \mathrm{pc}$ from the continuum source, we set up the inner radius of the broad line region according to the theoretical relation between the BLR inner radius and the monochromatic flux at $\lambda 550 \mathrm{~nm}$ for a $10^{44} \mathrm{erg} \mathrm{s}^{-1}$ AGN luminosity (Czerny et al. 2015). The BLR geometry is a flared disk with half-opening angle of $20^{\circ}$ from the equatorial plane (Marin et al. 2012). It is uniformly filled with electrons, and the Keplerian motion of the BLR is fixed to $4000 \mathrm{~km} \mathrm{~s}^{-1}$ (Netzer 1990). The radial optical depth in the $V$ band of the BLR region is set to 3 at the present stage but varied in the photoionizing continuum-dimming scenario. The outer radius of the BLR is set at the inner radius of the dusty torus, physically constrained by the dust sublimation radius. Following the monitoring observations made by Suganuma et al. (2006) in the optical and nearinfrared wave bands for a set of Seyfert type 1 galaxies, we chose a torus inner radius of $0.1 \mathrm{pc}$. The torus outer radius is fixed

\footnotetext{
2 http://www.stokes-program.info/
}

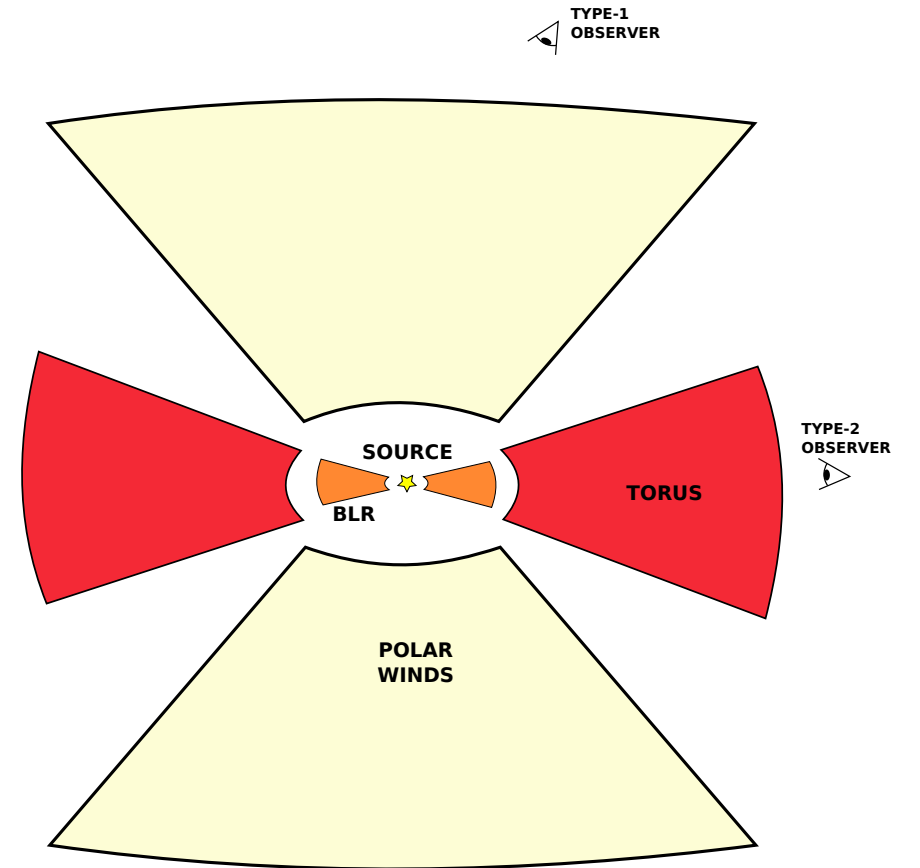

Fig. 1. Unscaled AGN model used in this paper. The central optical source (yellow star) irradiates an electron-filled broad line region (BLR, in orange) and a coplanar, more distant, dust-filled circumnuclear region (torus, in red). The source also irradiates torus-collimated, ionized, ejection winds along the polar axis (yellow). Quantitative details such as sizes, half-opening angles or composition are given in the text.

to $5 \mathrm{pc}$, following recent interferometric observations (see, e.g., Tristram et al. 2007). The half-opening angle of the circumnuclear region is set to $45^{\circ}$ and its optical depth is higher than 50 in the $V$ band. We note that the value of the torus half-opening angle also changed in the scenario, which explains the spectral variations of changing-look quasars by dust obscuration. Finally, the torus half-opening angle collimates a pair of conical ejection winds along the polar axes. The winds begin at a radial distance of $0.01 \mathrm{pc}$ from the central source and stop at $10 \mathrm{pc}$. The outflows are filled with electrons (Antonucci \& Miller 1985), and their radial optical depth is on the order of 0.1 (Marin et al. 2012). This AGN model was used to infer the optical linear continuum polarization of changing-look AGN according to the scenarios detailed in Sect. 1. For clarity, we focus our modeling on type 1 to type 1.9/2 transitions for the remainder of this paper.

\section{Results}

\subsection{AGN polarization and ionizing continuum dimming}

We present the results of our computations for the generic AGN model in Fig. 2. The top panel is the total optical flux normalized to the maximum flux observed at an inclination of $0^{\circ}$. For type 1 viewing angles (up to the torus horizon, i.e., $45^{\circ}$ ), the flux is almost constant, but suddenly drops at types 2 orientations. This transition is also visible in the middle and bottom panels, the continuum linear polarization degree and polarization position angle, respectively. At type 1 views, the degree of polarization is small $(<4 \%)$ and associated with a polarization angle of $90^{\circ}$ (parallel to the symmetry axis of the system). The polarization degree is null at perfect pole-on inclinations because of the symmetry of the system. The polarization degree then increases with inclination until the transition between type 1 and type 2 lines 


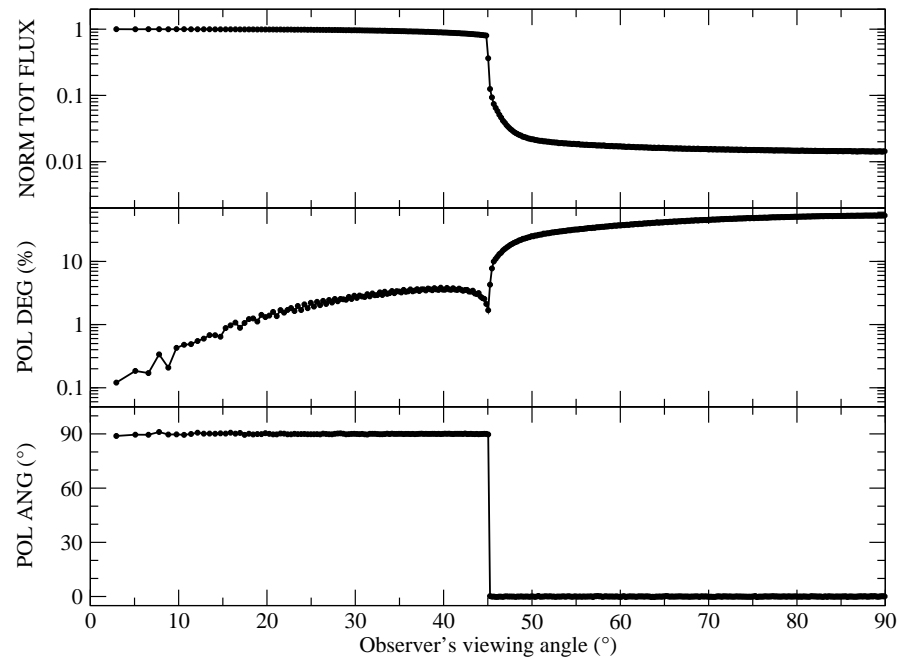

Fig. 2. Variation in optical total flux (normalized, top), optical linear polarization degree (middle), and polarization position angle (bottom) with respect to the inclination of the observer.

of sight. The polarization position angle rotates down to $0^{\circ}$ (perpendicular to the symmetry axis of the system), and the polarization degree increases to $54 \%$ at equatorial views. In the case of a dimming of the continuum source, the amount of radiation scattering of the BLR region will be smaller and the flux will drop. This will result in a lower normalized flux at all inclinations, and the lack of photoionizing radiation will prevent the emission of strong broad emission lines. However, this will have no effect on the intrinsic polarization degree and angle since they are relative quantities. For this interpretation, we expect to see no variations in the (linear continuum) polarization properties of the source.

\subsection{BLR-fading scenario}

In the case of a strong dimming (bolometric luminosity $\leq 10^{42} \mathrm{erg} \mathrm{s}^{-1}$ ), the work of Elitzur et al. (2014) suggests that the broad line emission region follows an evolutionary sequence that is directly related to the accretion rate of the compact source. If this is true, the BLR becomes less dense as the accretion rate decreases, and eventually disappears (Elitzur \& Shlosman 2006). To investigate this interpretation, we decreased the optical depth of the BLR from 3 to 0.01 to simulate the progressive disintegration of the region. Following Elitzur \& Shlosman (2006), the timescales for the BLR and torus disappearance is short, that is, a few Keplerian orbits. We note that in our modeling, we consider a strong coupling between the BLR and the electron-scattering disk predicted by Smith et al. (2002). The equatorial electron region is necessary to reproduce both the observed polarization position angle of type $1 \mathrm{AGN}$ and the intrinsic polarization of broadened lines. The electron disk is considered to be a continuous flow between the torus and the inner parts of the BLR (ibid). Smith et al. (2002) reported that the two regions were disconnected since their code only considered single scattering, but there is no physical reasons for a decoupling of the two regions when multiple scattering is enabled.

Figure 3 shows the normalized total flux at $\lambda 550 \mathrm{~nm}$, the polarization degree, and the polarization position angle of the AGN model as a function of the BLR optical depth $\tau$. The observer's inclination is fixed at a typical type 1 inclination angle of $30^{\circ}$ with respect to the symmetry axis of the model. At $\tau=3$, the degree of polarization is maximum (about 2.79\%)

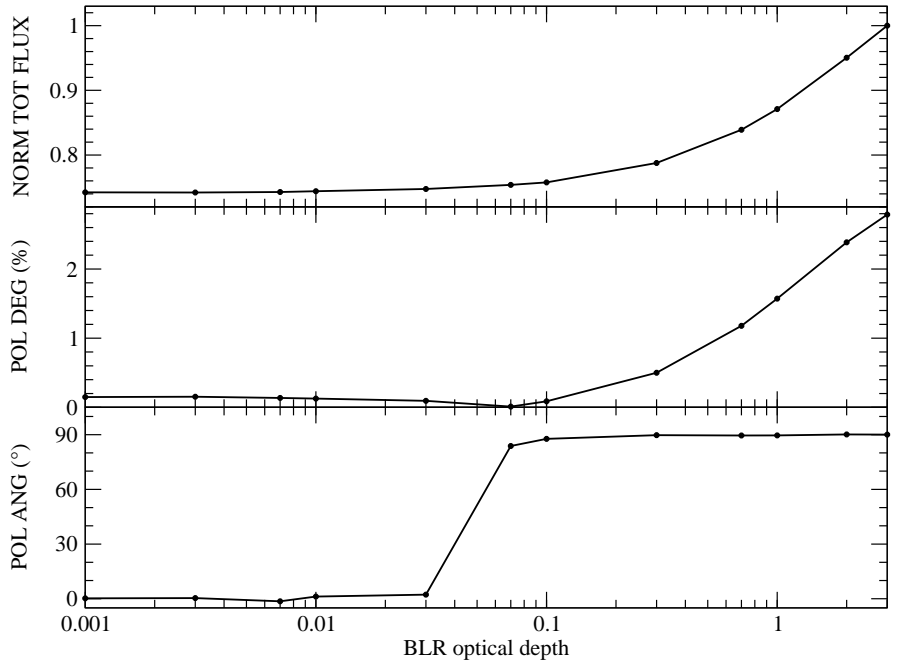

Fig. 3. Variation in optical total flux (normalized, top), optical linear polarization degree (middle), and polarization position angle (bottom) with respect to the gradual fading of the BLR region. The observer's inclination is set to $30^{\circ}$.

and the polarization position angle is equal to $90^{\circ}$. This corresponds to the expected polarization angle of type $1 \mathrm{AGN}$ and the relatively high degree of polarization obtained is due to the optical thickness of the BLR. The efficiency of the equatorial medium to intercept radiation and scatter it toward the observer is maximum. The resulting polarization degree, despite being diluted by the unpolarized central source seen by transmission through the wind, is thus higher than $1 \%$. For higher densities of the BLR, multiple scattering will start to depolarize radiation. However, when the BLR region starts to decrease in density because of a lower accretion rate, the electron equatorial scattering is weaker. The polarization degree thus decreases gradually with $\tau$. It reaches a minimum around $\tau=0.05$, where the polarization position angle rotates from $90^{\circ}$ to $0^{\circ}$. Equatorial scattering is then inefficient to produce the observed polarization angle of Seyfert type $1 \mathrm{~s}$ and the polarization degree remains below $0.15 \%$ for the lower optical depths of the BLR. The observed total flux of the AGN follows the same trend as the polarization degree, decreasing by $25 \%$ when the BLR is extremely optically thin. We thus see that when the changing-look nature of the few AGN observed is due to the disappearance of the BLR, we expect a strong diminution of the total flux. The polarization degree should also decrease, and an orthogonal flip of the polarization position angle is expected in the optical band.

\subsection{Varying the amount of dusty obscuration}

Figure 5 presents the results of our modeling for the alternative interpretation behind the changing-look nature of AGN. In this case, it is assumed that the amount of dust absorption along the observer's line of sight varies (Matt et al. 2003). To model it, we fixed the optical depth of the BLR to 3 and added a removable outer layer with an opening angle of $5^{\circ}$ at the top of the circumnuclear region that is blocking the view of a type 1.9/2 observer. When the dusty layer is at the top of the torus, the halfopening angle of the circumnuclear dust material changes from $45^{\circ}$ to $50^{\circ}$ (measured from the equatorial plane). Consequently, the half-opening angle of the polar winds is reduced by $5^{\circ}$ with respect to the model presented in the previous section. Its value is then fixed to $40^{\circ}$. The layer of dust intercepting the observer's 


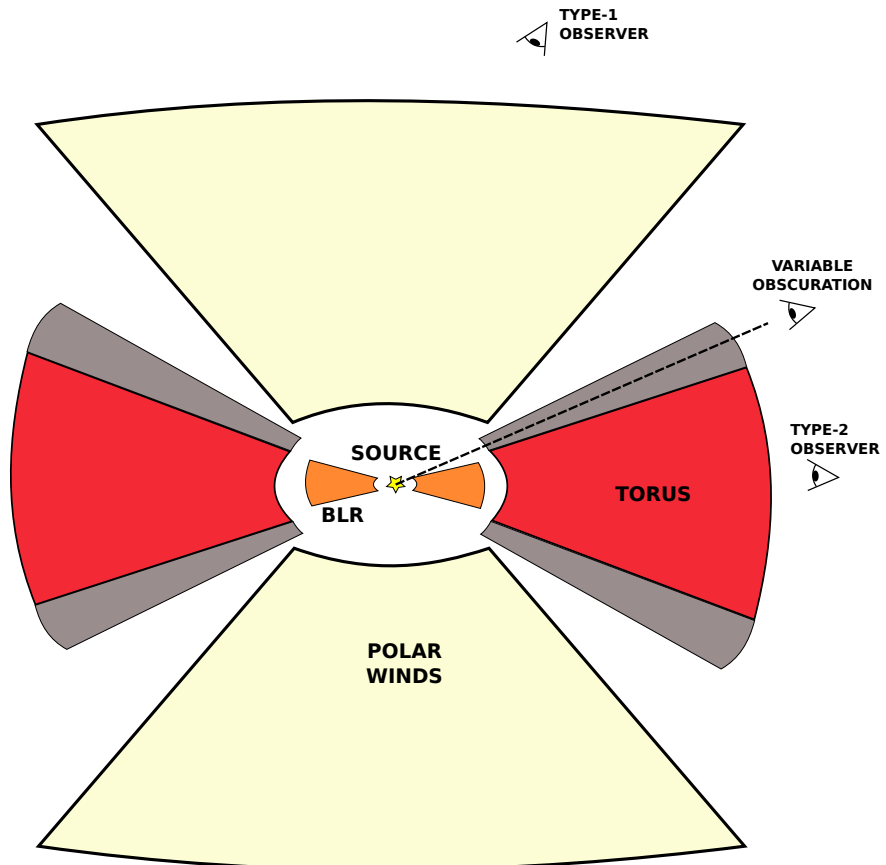

Fig. 4. Unscaled AGN model modified according to the variable torus obscuration interpretation. The outer layers of the torus (in gray) can be wiped out by an intense radiation field from the central region or when the torus is clumpy, by motion of individual clouds located on the observer's line of sight. The spectral type of the AGN thus changes from type $1.9 / 2$ to type 1 .

line of sight can be added or removed at will (see the gray region in Fig. 4). By shaving off the outer layers of the torus, we allow a type $1.9 / 2$ observer to have a direct (type 1) view of the central AGN engine without changing the nucleus orientation. This is represented by the dashed line in Fig. 4.

We examined the model from 30 observer inclinations equally distributed in cosine angle between $30^{\circ}$ and $60^{\circ}$. We focused on this range of inclinations as it represents the estimated transition angles between type 1 and type 2 AGN categories, slightly depending on the radio-loudness of the quasars (Baldi et al. 2013; Marin 2014, 2016; Sazonov et al. 2015; Marin \& Antonucci 2016). Figure 5 presents the results of the two scenarios: a), when the dusty layer is present at the top of the torus (in black); and b) when this layer is removed (in red). The top panel shows the difference in normalized total flux between the two scenarios as a function of the observer's inclination. At the inclinations where the observer's viewing angle crosses the removable layer of dust (shaded gray region in Figs. 4 and 5), the flux drops by a factor that is inclination dependent for a variable amount of obscuration. At maximum, the flux difference is a factor 40 between the unobscured (type 1) and obscured (type 1.9/2) model. The transition angle at which the two models change from type 1 to type 2 strongly depends on the presence or absence of the dusty layer. At a given inclination, the effect of variable obscuration onto the computed flux is evident. The lower flux observed in the type 1.9/2 scenario is due to radiation scattered onto the polar winds, plus a minor contribution of backscattering of photons onto the torus funnel opposite to the observer's side. This change in scattering geometry (from direct light to polar-scattered radiation) has a profound effect on the polarization degree and angle. For a given inclination within the gray area (where variable obscuration occurs), the difference in terms of optical continuum polarization for the two scenarios

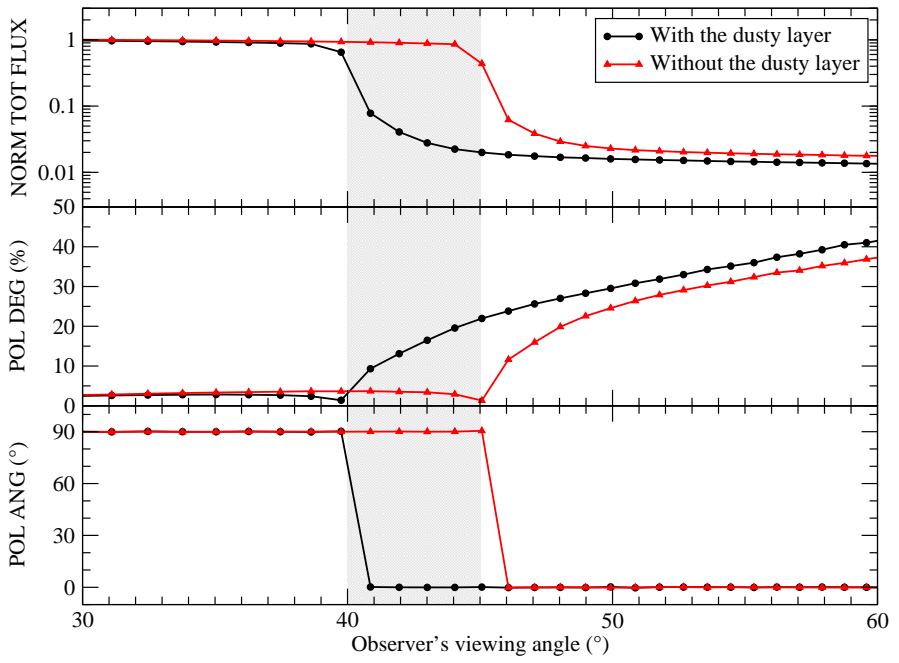

Fig. 5. Results of the two scenarios described in the text: with a dusty layer at the top of the torus (black dots) and when the layer is removed (red triangles). The top panel shows the normalized total flux, the middle panel shows the polarization degree, and the bottom panel presents the observed polarization position angle for the two cases. The gray box represents the angular region that is obscured by the removable dusty layer.

is large. When the line of sight toward the nucleus is uncovered, the polarization degree is only some percent, while it rises to $10-20 \%$ when the torus horizon is obscured with dust. The exact variation in polarization degree between the two states of the changing-look AGN depends on the inclination of the observer. Nevertheless, it is always a significant change that can be as high as $20 \%$. This increase in polarization is directly due to the different paths radiation has to follow to escape the type 1 and type 1.9/2 models. Additionally, the change in spectral type is systematically associated with an orthogonal rotation of the polarization position angle.

\section{Discussion}

The three interpretations (the ionizing continuum dimming, the BLR fading, and the variation in dusty obscuration scenarios) have very clear and distinctive features in total flux and polarimetry. In the first case, a change in continuum radiation due to a variation in accretion rate will not change the observed polarization properties of the AGN. When the accretion rate becomes insufficient to sustain the BLR (and torus), the total flux of the AGN should change by a factor $\sim 25 \%$ simply because the equatorial scattering that redirects photons towards the observer is very strong. With decreasing accretion rates onto the central supermassive black hole, the polarization degree also decreases as electron scattering inside the BLR becomes inefficient. The broad line emission will appear increasingly weaker, and the polarization position angle finally rotates by $90^{\circ}$. In the last case, the difference in total flux before and after a change of look is much higher. Up to $98 \%$ of the central flux is absorbed by the circumnuclear dusty medium. Radiation escapes from the AGN by scattering inside the polar outflows and thus carries a higher polarization degree as a result of the Thomson laws. The polarization position angle also rotates between the two spectral states. An increase of about $10-20 \%$ in polarization degree is expected for a changing-look quasar that alternates from a type 1 to a type 2 classification. 


\section{1. $J 1011+5442$}

The three interpretations are thus distinctively different, except for their polarization position angle, which might rotate in the last two cases. By monitoring the flux and polarization state of a sample of changing-look AGN candidates, it would be possible to easily distinguish the correct physical reason behind the spectral variations. However, we currently do not have archival large monitoring campaigns of AGN polarization. To overcome this lack of data, Hutsemékers et al. (2017) used a single-polarization measurement as a diagnostic tool of the changing-look nature of the quasar SDSS J101152.98+544206.4 (hereafter J1011+5442). The authors used the William Herschel telescope to measure the polarization of $\mathrm{J} 1011+5442$, a $z=0.246$ quasar with an absolute magnitude $M_{\mathrm{i}}=-22.87$ (Shen et al. 2011). Between 2002 and 2015, the blue continua and broad optical emission lines of $\mathrm{J} 1011+5442$ have been observed to decline (Runnoe et al. 2016), changing the optical classification of the quasar from type 1 to type 1.9. Hutsemékers et al. (2017) observed $\mathrm{J} 1011+5442$ in its faint state on February 19, 2017, and after correcting for the chromatic dependence of the half-waveplate zero-angle and instrumental polarization, found a linear polarization degree of $0.15 \pm 0.22 \%$. Knowing that the interstellar polarization toward $\mathrm{J} 1011+5442$ is expected to be on the order of $0.1 \%$, Hutsemékers et al. (2017) concluded that the polarization of the quasar is compatible with a null intrinsic polarization ${ }^{3}$. Based on their results and theoretical polarization arguments, the authors suggested that the quasar was seen at an inclination close to the pole and that the undetected polarization degree was proof of the lack of photoionizing continuum. The Monte Carlo simulations achieved in this paper clearly prove the correctness of their conclusion: if $\mathrm{J} 1011+5442$ changed its spectral state because of additional equatorial dust obscuration along the observer's line of sight, its intrinsic degree of polarization should be on the order of $10-20 \%$ and thus easily detectable against interstellar polarization. Thanks to our computations, we can go two steps further. First, if the polarization degree of the quasar is on the order of $0.15 \pm 0.22 \%$, its nucleus inclination is strictly inferior to $9^{\circ}$ (see Fig. 2). This is a conservative estimation of the inclination since it does not depend on the exact half-opening angle or optical depths of the model components, but rather on the axisymmetry of the unified model itself (Marin et al. 2012). Second, Fig. 3 from Runnoe et al. (2016) shows that the decrease in flux at $\lambda 550 \mathrm{~nm}$ between the two epochs is approximately $35 \%$. This drop in flux is similar to what we found in Sect. 3 when the BLR fades away (25\%). The difference can be easily compensated for by an intrinsic dimming of the continuum source and/or a modification of the geometrical configuration of the broad emission line region (the half-opening angle and optical depth of the BLR being the two critical parameters here). However, this drop in flux is clearly not compatible with the $98 \%$ diminution expected in the scenario where dust clouds from the torus genuinely block radiation from the central engine.

\subsection{Polarization reverberation mapping}

As has been mentioned in Hutsemékers et al. (2017), scattering in AGN is considered occur at up to a few parsecs. Additionally, the amount of reprocessing events is inclination dependent, as shown in Fig. 2 and explained in Sect. 3.1. Hence, if the central continuum source suddenly suffers a strong dimming,

\footnotetext{
3 The polarization position angle of $\mathrm{J} 1011+5442$ could not be estimated with reasonable accuracy (Hutsemékers et al. 2017).
}

polarization from the polar outflows that extend over $10 \mathrm{pc}$ (Capetti et al. 1995) is expected to last up to $\sim 32 \mathrm{yr}$ after the continuum change. A polarimetric echo of the past core activity will remain visible in the extended structures of the AGN, as expected in the case of the Galactic center in the X-ray band (Churazov et al. 2002; Marin et al. 2014, 2015b). The observed polarization properties of an AGN, if integrated over the whole structure, will be affected. Since the timescale of changes of look is on the order of a few years (MacLeod et al. 2016), this might affect the proposed polarization diagnostics unless high angular resolution polarization maps are available for nearby objects (as for the case of NGC 1068, which was observed using the Spectro-Polarimetric High-contrast Exoplanet REsearch instrument - SPHERE - on the Very Large Telescope, see Gratadour et al. 2015).

This is where the polarization reverberation mapping technique is the most valuable. Gaskell et al. (2012) demonstrated how the polarization variability in NGC 4151 can be used to probe the size and structure of scattering regions. Between 1997 and 2003, the type 1 radio-quiet AGN NGC 4151 has shown variations of an order of magnitude in its optical polarized flux while its polarization position angle remained constant. Since the sizes of the different scattering regions in AGN span several orders of magnitude, the time delay we measure is directly related to the geometry of the system. Scattering inside the BLR produces a polarization angle parallel to the radio axis of the system, and its temporal delay is shorter than photons that have scattered onto the polar outflows, where the scattering-induced polarization angle is perpendicular. It follows that polarization reverberation mapping can precisely locate where scattering occurs. In addition, if temporal changes in the wavelength dependence of polarization across broad emission lines are detected, this could imply a change in the BLR and scatterer geometry (Shoji et al. 2005). This effect would be enhanced for increasing nucleus inclinations, and the two scenarios discussed in this paper could in principle be distinguished thanks to polarization reverberation mapping (Rojas Lobos et al. 2017; Marin et al., in prep.).

\section{Conclusions}

We have investigated the different interpretations behind the changing-look nature observed for a few AGN. The first model predicts that the disappearance of the broad emission line and the decrease in flux are due to the vanishing of the broad emission line region, which is linked to a decrease of the black hole accretion rate. If the accretion rate becomes too low, the BLR itself can progressively disappear since mass accretion can no longer sustain the required column densities. The last model explains the same spectral changes with a variation in the obscuration of the observer's viewing angle that is grazing the torus horizon. Either the outer layers of the torus are puffed-up, or clouds intercept the line of sight. For the first time, both models were investigated using radiative transfer Monte Carlo calculations, and their optical flux and polarization signals were found to be distinctively different. If the spectral variations are due to a lack of photionizing radiation, the flux should drop accordingly but no variation of the polarization properties are expected, since they are relative quantities and do not depended on the amount of photon scattering inside the BLR. In the case of a progressive disappearance of the BLR, a $\sim 25 \%$ decrease in total flux and a polarization degree $<0.1 \%$ are expected. Finally, if variable obscuration is the correct scenario, the total flux should be reduced by about a factor 40 and the polarization degree should be at 
least $10 \%$ higher. In the last two cases, the polarization position angle should rotate by $90^{\circ}$.

We applied our results to the $\mathrm{J} 1011+5442$ quasar investigated by Hutsemékers et al. (2017) and found strong evidence for the correctness of their interpretation. We extended the polarimetric investigation of the authors by estimating the nucleus inclination of the quasar $\left(<9^{\circ}\right)$. Our computations show that the total flux variation of the quasar over a decade is also consistent with their primary conclusion: the broad emission line region of J1011+5442 switched off between 2002 and 2015. The same conclusion was postulated by LaMassa et al. (2015) and MacLeod et al. (2016) based on specific and systematic photometric searches for changing-look quasars. For the first time, we here provide detailed computations of the expected flux attenuation and optical polarization expected from all the scenarios.

Our paper clearly shows that distinguishing between the various physical interpretations is easy. New optical polarimetric observations of AGN showing a changing-look behavior will immediately tell whether the change is due to an intrinsic dimming of the ionizing continuum source, a BLR disappearance, or a variation in line of sight obscuration. Prior polarimetric measurements are not vital since the actual intrinsic polarization degree should be significant enough to tell the two last models apart (null or $<0.1 \%$ polarization in the first case; high, $\geq 10 \%$ polarization in the other case). Archival polarimetric information about the polarization angle and degree would be beneficial to determine whether the spectral change is simply due to a lack of photoionizing radiation (no variations in polarization between the two epochs) or a progressive disappearance of the BLR (decrease of polarization degree and rotation of the polarization position angle). Coupling the polarimetric measurements with past photometric data would greatly facilitate the interpretation, since the change in flux level is also model dependent. We therefore advocate systematic polarimetric observations of changing-look AGN in order to fully understand their true nature.

Acknowledgements. The author would like to acknowledge the anonymous referee for useful comments that improved the quality of the paper.

\section{References}

Antonucci, R. R. J., \& Miller, J. S. 1985, ApJ, 297, 621

Baldi, R. D., Capetti, A., Buttiglione, S., Chiaberge, M., \& Celotti, A. 2013, A\&A, 560, A81

Binney, J., \& Merrifield, M. 1998, Galactic Astronomy (Princeton: Princeton University Press)

Brindle, C., Hough, J. H., Bailey, J. A., et al. 1990, MNRAS, 244, 577
Capetti, A., Macchetto, F., Axon, D. J., Sparks, W. B., \& Boksenberg, A. 1995, ApJ, 452, L87

Churazov, E., Sunyaev, R., \& Sazonov, S. 2002, MNRAS, 330, 817

Code, A. D., Meade, M. R., Anderson, C. M., et al. 1993, ApJ, 403, L63

Cohen, R. D., Puetter, R. C., Rudy, R. J., Ake, T. B., \& Foltz, C. B. 1986, ApJ, 311, 135

Czerny, B., Modzelewska, J., Petrogalli, F., et al. 2015, Adv. Space Res., 55, 1806

Elitzur, M., \& Ho, L. C. 2009, ApJ, 701, L91

Elitzur, M., \& Shlosman, I. 2006, ApJ, 648, L101

Elitzur, M., Ho, L. C., \& Trump, J. R. 2014, MNRAS, 438, 3340

Gaskell, C. M. 2009, New Astron. Rev., 53, 140

Gaskell, C. M., Goosmann, R. W., Merkulova, N. I., Shakhovskoy, N. M., \& Shoji, M. 2012, ApJ, 749, 148

Goodrich, R. W. 1989, ApJ, 340, 190

Goosmann, R. W., \& Gaskell, C. M. 2007, A\&A, 465, 129

Gratadour, D., Rouan, D., Grosset, L., Boccaletti, A., \& Clénet, Y. 2015, A\&A, 581, L8

Hutsemékers, D., Agís González, B., Sluse, D., Ramos Almeida, C., \& Acosta Pulido, J.-A. 2017, A\&A, 604, L3

LaMassa, S. M., Cales, S., Moran, E. C., et al. 2015, ApJ, 800, 144

MacLeod, C. L., Ross, N. P., Lawrence, A., et al. 2016, MNRAS, 457, 389

Marin, F. 2014, MNRAS, 441, 551

Marin, F. 2016, MNRAS, 460, 3679

Marin, F., \& Antonucci, R. 2016, ApJ, 830, 82

Marin, F., \& Goosmann, R. W. 2014, in SF2A-2014: Proc. Annual Meeting of the French Society of Astronomy and Astrophysics, eds. J. Ballet, F. Martins, F. Bournaud, R. Monier, \& C. Reylé, 103

Marin, F., \& Grosso, N. 2017, ApJ, 835, 283

Marin, F., Goosmann, R. W., Gaskell, C. M., Porquet, D., \& Dovčiak, M. 2012, A\&A, 548, A121

Marin, F., Karas, V., Kunneriath, D., \& Muleri, F. 2014, MNRAS, 441, 3170

Marin, F., Goosmann, R. W., \& Gaskell, C. M. 2015a, A\&A, 577, A66

Marin, F., Muleri, F., Soffitta, P., Karas, V., \& Kunneriath, D. 2015b, A\&A, 576, A19

Matt, G., Guainazzi, M., \& Maiolino, R. 2003, MNRAS, 342, 422

Miller, J. S., \& Antonucci, R. R. J. 1983, ApJ, 271, L7

Netzer, H. 1990, in Active Galactic Nuclei, eds. R. D. Blandford, H. Netzer, L. Woltjer, T. J.-L. Courvoisier, \& M. Mayor, 57

Osterbrock, D. E. 1977, ApJ, 215, 733

Pringle, J. E., \& Rees, M. J. 1972, A\&A, 21, 1

Ricci, C., Bauer, F. E., Arevalo, P., et al. 2016, ApJ, 820, 5

Risaliti, G., Maiolino, R., \& Bassani, L. 2000, A\&A, 356, 33

Rojas Lobos, A., Goosmann, R. W., Marin, F., \& Savic, D. 2017, A\&A submitted

Rowan-Robinson, M. 1977, ApJ, 213, 635

Runnoe, J. C., Cales, S., Ruan, J. J., et al. 2016, MNRAS, 455, 1691

Sazonov, S., Churazov, E., \& Krivonos, R. 2015, MNRAS, 454, 1202

Shakura, N. I., \& Sunyaev, R. A. 1973, A\&A, 24, 337

Shen, Y., Richards, G. T., Strauss, M. A., et al. 2011, ApJS, 194, 45

Shoji, M., Gaskell, C. M., \& Goosmann, R. W. 2005, in AAS Meeting Abstracts 207, BAAS, 37, 1420

Simpson, C. 2005, MNRAS, 360, 565

Smith, P. S., Schmidt, G. D., Allen, R. G., \& Hines, D. C. 1997, ApJ, 488, 202

Smith, J. E., Young, S., Robinson, A., et al. 2002, MNRAS, 335, 773

Suganuma, M., Yoshii, Y., Kobayashi, Y., et al. 2006, ApJ, 639, 46

Tristram, K. R. W., Meisenheimer, K., Jaffe, W., et al. 2007, A\&A, 474, 837

Zirbel, E. L., \& Baum, S. A. 1998, ApJS, 114, 177 